\title{
miRNA analysis of formalin-fixed squamous cell carcinomas of the tongue is affected by age of the samples
}

\author{
M. RENTOFT ${ }^{1}$, J. FAHLÉN ${ }^{2}$, P.J. COATES ${ }^{3}$, G. LAURELL ${ }^{4}$, B. SJÖSTRÖM ${ }^{4}$, P. RYDÉN ${ }^{5}$ and K. NYLANDER ${ }^{1}$ \\ ${ }^{1}$ Department of Medical Biosciences/Pathology, Building 6M, Umeå University, SE $90185 ;{ }^{2}$ Department of Statistics, \\ Umeå University, 90187 Umeå, Sweden; ${ }^{3}$ Division of Medical Sciences, University of Dundee, Ninewells Hospital \\ and Medical School, Dundee, UK; Departments of ${ }^{4}$ Clinical Sciences/Otorhinolaryngology, Umeå University Hospital \\ and ${ }^{5}$ Mathematics and Mathematical Statistics, Umeå University, SE 90187 Umeå, Sweden
}

Received August 6, 2010; Accepted September 16, 2010

\section{DOI: 10.3892/ijo_00000824}

\begin{abstract}
Global miRNA expression arrays were used for analysis of 836 miRNAs in formalin-fixed paraffin-embedded samples from 21 tongue cancer patients and 8 controls. Samples had been stored for one to eleven years. Results separated tumour samples from controls, however, the largest variation was correlated to sample storage time, detectable already after one year. With the use of a linear regression model we could adjust for the storage-dependent effect, leading to the identification of 54 differentially expressed miRNAs in tongue cancer, compared to 16 when using standard normalization, including up-regulation of a novel miRNA, miR-424.
\end{abstract}

\section{Introduction}

MicroRNAs (miRNAs) were discovered in C.elegans (Caenorhabitis elegans) over a decade ago and were initially believed to be a species-specific phenomenon $(1,2)$. Today there are $>800$ confirmed human-specific miRNAs registered in mirBASE, with more being added every year (3). miRNAs are small regulatory RNAs ( $\sim 22 \mathrm{nt}$ long) that in a sequencedependent manner bind to and regulate coding RNA at the posttranscriptional level. miRNAs have been implicated in a number of cellular processes and disease states (e.g. cell development, cell proliferation, apoptosis and cancer) and might target as many as one third of the genes in the human genome (4-6). miRNAs demonstrate a number of properties that could make them ideal for tumour prediction and classification $(7,8)$ and changes in only a few miRNAs could have considerable biological effects due to the large number of genes each miRNA is predicated to target (9-11). Microarray technologies have assisted the resolution of many questions

Correspondence to: Dr K. Nylander, Department of Medical Biosciences/Pathology, Building 6M, 2nd floor, Umeå University, SE 90185 Umeå, Sweden

E-mail: karin.nylander@medbio.umu.se

Key words: miRNA, FFPE, tongue carcinoma, storage, normalization previously impossible to answer and the technique has recently made its way into daily clinical practice with the MammaPrint gene expression signature assay (12).

The standard method for preserving tissue from patients for diagnostic purpose is fixation in formalin followed by embedding in paraffin (FFPE) and millions of such FFPE blocks are stored around the world. The use of this type of samples in large scale gene expression experiments gives the possibility of including an extensive number of patients, with long-term follow-up (13). While formalin fixation is an excellent method for preserving the architecture of the tissue for histological investigation, it causes degradation and modification of nucleic acids making it difficult to study gene expression levels in FFPE samples $(14,15)$. miRNAs are hypothesised to be more stable than mRNA in FFPE samples due to their small size and secondary structure, and a number of studies have shown that miRNA profiles from formalinfixed and fresh-frozen tissue correlate well (16-18), and some have reported decreased ability to detect certain miRNAs with prolonged storage time $(17,19)$.

The aim of this study was to investigate the expression of 836 miRNAs in FFPE samples of squamous cell carcinoma (SCC) of the tongue in comparison with normal tongue tissue, using miRNA expression arrays. Data showed that the largest variation was correlated to storage time of the samples, a bias that standard normalization methods could not remove. By applying a novel normalization method we detected 54 miRNAs to be significantly differentially expressed between tumour and control tissue, in contrast to the 16 differentially expressed miRNAs identified by the standard analysis method.

Among the 54 miRNAs the consistent up-regulation of a novel miRNA in tumour samples, miR-424 and two known miRNAs, miR-21 and miR-203 was confirmed in a set of frozen tissue samples. These observations demonstrate that storage time of FFPE samples leads to loss of information, which, however, can be compensated for, allowing biologically relevant changes to be identified.

\section{Materials and methods}

Samples. FFPE samples from 21 patients with SCC on the tongue and eight patients with benign lesions from the same site (control tissue) as well as five paired fresh-frozen (FF) 
Table I. Patient and sample data.

\begin{tabular}{|c|c|c|c|}
\hline Sample & Patient age & Group & Storage time (year) \\
\hline 42 & 76 & Tumour & 0.8 \\
\hline 32 & 34 & Tumour & 1.3 \\
\hline 13 & 38 & Tumour & 1.3 \\
\hline 39 & 59 & Tumour & 1.7 \\
\hline 1 & 74 & Tumour & 2.1 \\
\hline 14 & 52 & Tumour & 2.1 \\
\hline 6 & 74 & Tumour & 2.3 \\
\hline 41 & 60 & Tumour & 2.4 \\
\hline 17 & 40 & Control & 2.8 \\
\hline 18 & 16 & Control & 2.8 \\
\hline 11 & 58 & Tumour & 3.0 \\
\hline 16 & 40 & Control & 3.2 \\
\hline 30 & 40 & Tumour & 3.4 \\
\hline 4 & 39 & Tumour & 4.0 \\
\hline 15 & 35 & Control & 4.3 \\
\hline 21 & 41 & Control & 4.7 \\
\hline 31 & 65 & Tumour & 4.7 \\
\hline 38 & 72 & Control & 4.7 \\
\hline 37 & 38 & Control & 5.7 \\
\hline 43 & 80 & Tumour & 6.5 \\
\hline 27 & 79 & Tumour & 6.7 \\
\hline 23 & 71 & Control & 7.0 \\
\hline 8 & 38 & Tumour & 7.0 \\
\hline 36 & 29 & Tumour & 7.4 \\
\hline 26 & 31 & Tumour & 8.0 \\
\hline 25 & 27 & Tumour & 8.9 \\
\hline 35 & 40 & Tumour & 9.8 \\
\hline 29 & 36 & Tumour & 10.8 \\
\hline $24 b$ & 30 & Tumour & 11.2 \\
\hline
\end{tabular}

samples from tongue SCC and adjacent clinically normal mucosa (control tissue) were available for analysis. Of the 21 patients with FFPE samples, 11 were $<40$ years of age and 10 were $>50$ years of age. The mean age for the whole group was 39 years. Samples had been collected during 11 years and the majority (19 out of 29) processed at the Pathology Lab at Norrland's University Hospital in Umeå. Patient and sample data are summarized in Table I. The study was approved by the local Ethics Committee (dnr 01-210; dnr 08003M).

$R N A$ extraction. FFPE samples were cut into $5-\mu \mathrm{m}$ sections, and ten sections were used for RNA extraction, giving a total tissue area of approximately one $\mathrm{cm}^{2}$. RNA was isolated using the High Pure miRNA isolation kit (Roche Diagnostics $\mathrm{GmbH}$, Mannheim, Germany). Samples were eluted in $100 \mu 1$ elution buffer and concentration and purity of the samples measured spectrophotometrically using a Nanodrop ND-1000 spectrophotometer. RNA from the fresh-frozen, FF, samples was extracted using the trizole method and stored at $-80^{\circ} \mathrm{C}$ until use.
Table II. Description of the normalization procedures.

\begin{tabular}{lccc}
\hline Name & $\begin{array}{c}\text { Background } \\
\text { correction }\end{array}$ & $\begin{array}{c}\text { Dye- } \\
\text { normalization }\end{array}$ & $\begin{array}{c}\text { Between array } \\
\text { normalization }\end{array}$ \\
\hline Normalization 1 & No & MA-loess & No \\
Normalization 2 & Yes & MA-loess & No \\
Normalization 3 & No & MA-loess & MAD \\
Normalization 4 & No & Quantile & No \\
\hline
\end{tabular}

Microarray hybridization. miRNA expression profiling was performed using a two-colour microarray platform (Exiqon, Vedbak, Denmark) where each sample was hybridized together with a common reference consisting of a mixture of all FFPE samples. Labelling was performed using the miRCURY ${ }^{\mathrm{TM}}$ LNA Array power labelling kit. Hy3-labeled samples were mixed with Hy5-labeled reference RNA and hybridized to the miRCURY LNA arrays, version 11.0 which contains capture probes targeting all miRNAs in the miRBASE version 12.0 in four replicate spots. The miRCURY LNA array microarray slides were scanned using the Agilent G2565BA Microarray Scanner System (Agilent Technologies, Inc., USA) and the image analysis was carried out using the ImaGene 8.0 software (BioDiscovery, Inc., USA).

Data pre-processing. Array data were initially normalized using four different standard procedures. All normalizations included three steps; background correction, dye-normalization and array normalization $(20,21)$. In order to normalize across arrays, data from each array was multiplied by a scaling factor based on the median absolute deviation (MAD). The four normalizations are described in Table II. Unless otherwise stated, normalization 1 was used in all analyses and in the figures presented.

Evaluation of storage time effect. Unsupervised hierarchical clustering, using the Manhattan distance and average linkage (22), as well as principal component analysis (PCA) (23) were used to visualize the normalized data set. To characterize the storage time-dependent variation found, linear regression was applied. For each miRNA and array the log-ratios between the aggregated Hy3 (tumour) and Hy5 (control) channel intensities were calculated. Henceforth these log-ratios are referred to as M-values. The average of the logarithmic transformed Hy3 and Hy5 intensities are referred to as A-values. To characterize the storage time-dependent variation observed, linear regression was applied to estimate the dependency between storage time and the normalized Mvalues. To reduce data complexity only tumour samples were included in this part of the analysis. The M-value of the $i$ th miRNA on the $j$ th array was modelled as:

$$
\mathrm{M}_{\mathrm{ij}}=\alpha_{\mathrm{i}}+\beta_{\mathrm{i}}^{*} \mathrm{t}_{\mathrm{j}}+\varepsilon_{\mathrm{ij}}
$$

where $\alpha$ is the intercept and the storage time. Here, the error term $\varepsilon_{\mathrm{ij}}$ is assumed to be independent and normally distributed with zero mean and constant variance. The regression coefficients were estimated by the method of least squares. 
A

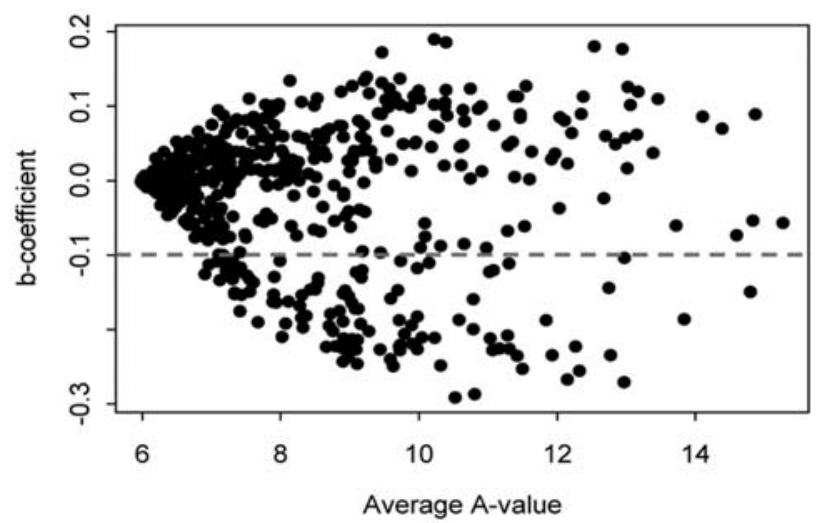

C

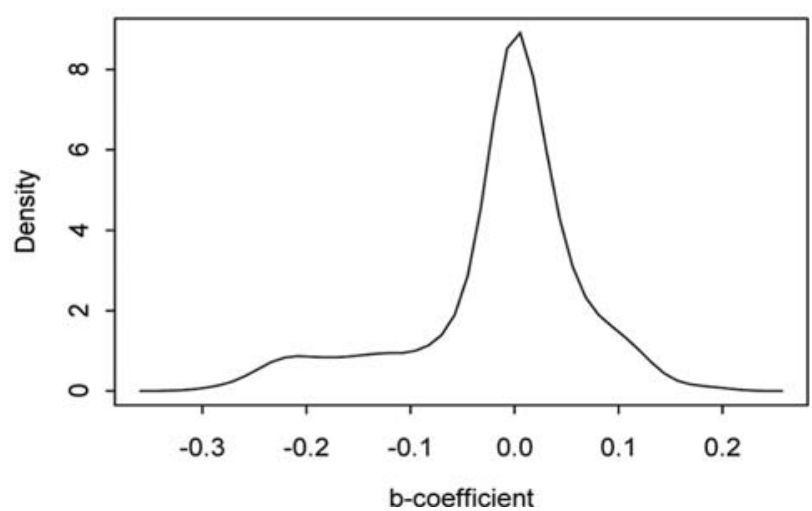

B

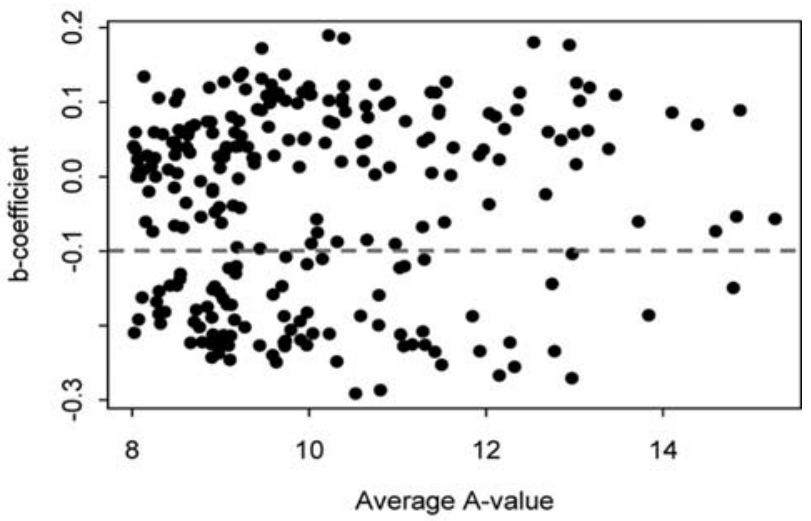

D

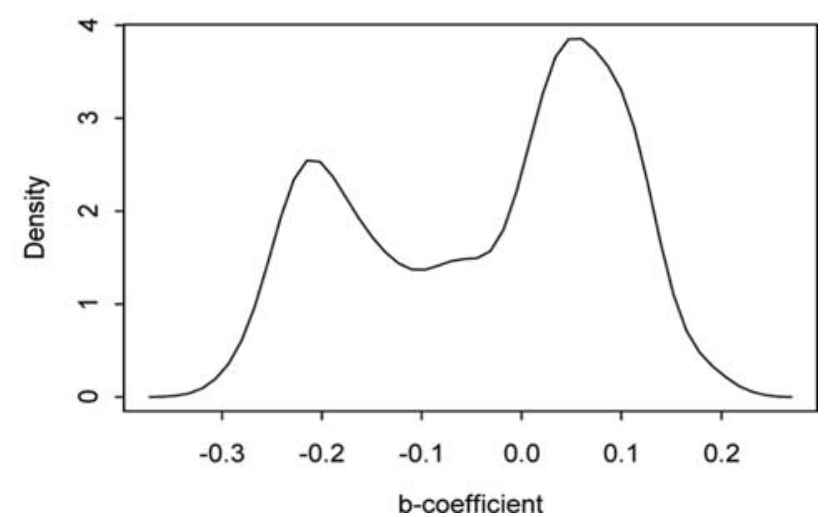

Figure 1. The b-coefficients against the average A-values (upper graphs) and density plots of the b-coefficients (lower graphs). Graph A and C include all miRNAs, while graph B and D include moderately and highly expressed miRNAS (average A-values $>8$ ). The b-coefficients of the moderately and highly expressed miRNAs are roughly separated into two groups, $>-0.1$ and $<-0.1$.

The estimated $\beta_{\mathrm{i}}$-coefficients, denoted b-coefficients, are estimates of the effect the storage time have on the miRNAs and were summarized in two graphs; where the b-coefficients were plotted against the average A-values and in a density plot (Fig. 1). The figure shows that the miRNAs roughly divide into two groups, suggesting that the storage time effect is miRNA dependent. In order to test if the observed groups could have occurred by chance a one-sample proportion test was applied.

Additional normalization procedure compensating for storage time. Linear regression was used to perform an additional normalization step compensating for storage time and resulting in M-values that were corrected for storage time (Mcorr). The normalization procedure was performed in three steps: 1) The raw data were normalized according to method 1 in Table II. 2) The M-values for the $i$ th miRNA on the $j$ th array were modelled according to:

$$
M_{i j}=\alpha_{i}+\beta_{i}^{*} t_{j}+\gamma_{i}^{*} z_{j}+\varepsilon_{i j}
$$

where $\mathrm{t}$ is the samples storage time and $\mathrm{z}$ an indicator variable describing if the sample came from a tumour or control tissue. The parameters in [2] were estimated using the method of least squares. 3) Corrected M-values (Mcorr) were obtained by subtracting the estimated storage time effect according to:

$$
\mathrm{M}_{\mathrm{ij}} \mathrm{corr}=\mathrm{M}_{\mathrm{ij}}-\mathrm{b}_{\mathrm{i}} \mathrm{t}_{\mathrm{j}}
$$

Note that, the variable $\mathrm{z}$ in equation [2] is needed to avoid subtracting the effect of interest, i.e. the effect between tumour and control samples.

Identification of significantly differentially expressed miRNAs. Gene lists with significantly differentially expressed genes were obtained using a regularized t-test (24) and the corresponding p-values were corrected for multiple testing by Bonferroni correction. The significance threshold was set at 0.05 .

Confirmation using $q$-PCR. q-PCR reactions were performed on a selection of miRNAs (SNORD48, miR-203, miR-21 and miR-424) using the miRCURY LNA ${ }^{\mathrm{TM}}$ Universal RT microRNA PCR system (Exiqon) according to the manufacturer's instructions. Briefly, $20 \mathrm{ng}$ of total RNA was incubated at $42^{\circ} \mathrm{C}$ for $60 \mathrm{~min}$ in the cDNA synthesis reaction. The cDNA was subsequently diluted 80 times and $8 \mu 1$ were used for each PCR reaction. All PCR reactions were analyzed in duplicate using a 7900HT real-time PCR system (Applied 


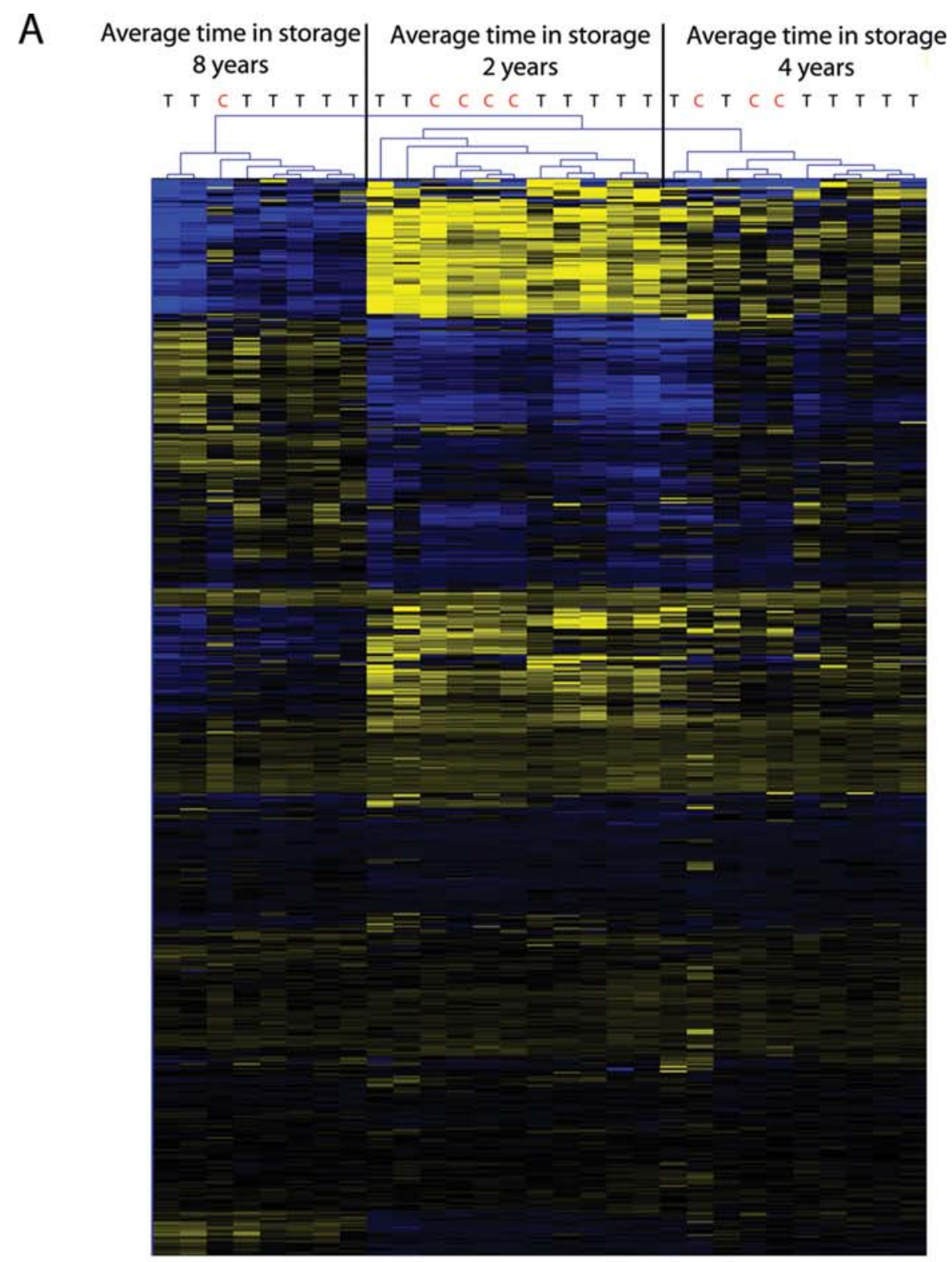

B

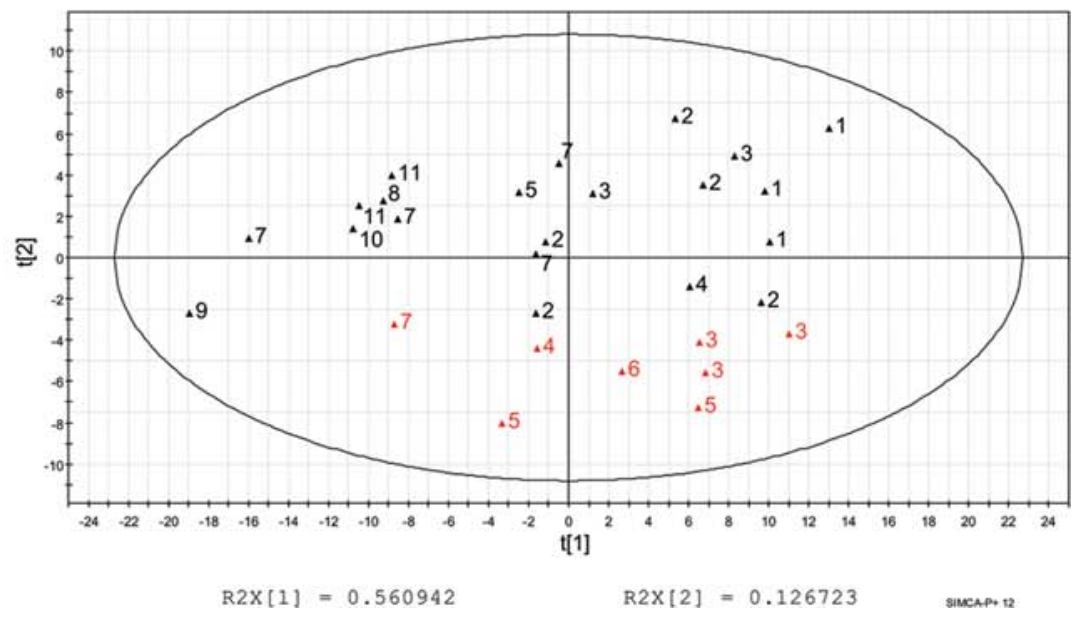

Figure 2. Initial visualization of array data. (A) Samples were clustered using unsupervised hierarchical clustering resulting in three main clusters. Samples are denoted as tumour (T) or control (C). The average time a sample has been stored is found above each cluster. (B) Score plot from PCA analysis showing first (t1) and second component (t2). The numbers denote time in storage in years and control samples are found in read while tumour samples are black. 
Table III. miRNAs significantly changed in SCC of the tongue (using data normalized with the standard method).

\begin{tabular}{llll}
\hline Up-regulated miRNA & p-value & Down-regulated miRNA & p-value \\
\hline hsa-miR-659 & $6,06 \mathrm{E}-03$ & hsa-miR-617 & $1,18 \mathrm{E}-03$ \\
hsa-miR-146b-3p & $8,53 \mathrm{E}-03$ & hsa-miR-29b-2* & $1,52 \mathrm{E}-03$ \\
hsa-miR-1301 & $1,09 \mathrm{E}-02$ & hsa-miR-132* & $8,44 \mathrm{E}-03$ \\
hsa-miR-665 & $1,28 \mathrm{E}-02$ & hsa-miR-548b-5p & $1,66 \mathrm{E}-02$ \\
hsa-miR-142-5p & $1,88 \mathrm{E}-02$ & hsa-miR-509-5p & $2,01 \mathrm{E}-02$ \\
hsa-miR-7 & $2,00 \mathrm{E}-02$ & hsa-miR-22* & $4,58 \mathrm{E}-02$ \\
hsa-miR-142-3p & $2,90 \mathrm{E}-02$ & & \\
hsa-miR-21* & $3,31 \mathrm{E}-02$ & & \\
hsa-miR-936 & $3,59 \mathrm{E}-02$ & & \\
hsa-miR-206 & $4,34 \mathrm{E}-02$ & & \\
\hline
\end{tabular}

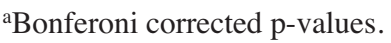

Biosystems, Foster City, CA, USA). SNORD48 was used as endogenous control and data analyzed using the $\Delta \Delta \mathrm{CT}$ method.

\section{Results}

RNA extraction and array hybridization. RNA for array analysis was extracted from 29 FFPE blocks corresponding to patient tissue from 21 tongue SCC and 8 non-malignant controls. No correlation between total amount of extracted RNA measured and age of the sample was seen. Samples from normal tongue tissue generated lower yield of total RNA irrespective of storage time. MiRNAs with low quality data (i.e. a large percentage missing values) were removed, reducing the original 836 miRNAs to 808 .

Visualization of array data. The primary visualization of the normalized data using unsupervised hierarchical clustering resulted in a dendrogram where controls and tumour samples spread out over three main clusters depending on sample storage time (Fig. 2A). Principal component analysis (PCA) confirmed that this clustering was highly correlated to a difference in storage time (Fig. 2B). The storage time-dependent effect was apparent already after one year in storage, and accounted for $56 \%$ of the total variation, while the second largest variation which distinctly distinguished tumour samples from control samples accounted for $13 \%$ of the total variation. Proceeding with the standard analysis, including normalization 1 and the regularized t-test (24), 16 miRNAs were identified as significantly differently expressed between tumour and control samples (Table III).

Characterization of the effect of storage time. As a systematic storage time-dependent variation (bias) within the data interferes with downstream data analysis, this variation was further characterized. The analysis showed that the 808 miRNAs divided into two rather distinct groups, one with increasing expression over time and the other with decreasing expression. This pattern was unlikely to be caused by chance and did not disappear until only samples stored less than a year were included in the analysis. Initially the linear regression model (1) was used to estimate the dependency between the miRNAs M-values (normalized log-ratios) and their storage times. As a result, a b-coefficient was obtained for each miRNA. The b-coefficient is an estimate of the effect storage time has on the M-value. For example a b-coefficient of -0.1 would mean that the M-value of the miRNA changes $-0.1^{*} 10=-1.0$ over 10 years. A change of the M-value with -1.0 translates into halving the log-ratio of the fresh sample. When plotting the b-coefficients against the average logintensity (A-value) the data should cluster around zero if storage time has no effect. However, as can be seen in Fig. 1, the miRNAs roughly separate into two groups $(b>-0.1$ and $\mathrm{b}<-0.1)$. This is especially apparent for moderately and highly expressed miRNAs (average $A>8$ ). The probability that the observed groups could have occurred by chance was very low $(\mathrm{p}<0.000001)$ suggesting that the storage time effect is miRNA dependent. This is important, since it implies that no standard normalization will work and that the miRNAs' data must be normalized individually. Removing the oldest samples one by one and recalculating the b-coefficients resulted in a similar separation of the miRNAs until only samples stored less than one year were included.

Identification of differentially expressed genes from data corrected for storage time. The novel normalization step was applied to all data in order to remove the bias and reduce the variance introduced by storing FFPE-samples over time. The normalized data set was again visualized using PCA and hierarchical clustering showing that the correlation to storage time had been adjusted, or at least reduced while the differences between control and cancer patients remained.

With the novel normalization, 54 miRNAs were identified as significantly differently expressed between tumour and control samples (Table IV), compared to the 16 miRNAs identified in the standard normalized data. All 16 miRNAs that were identified in the traditional approach were also found when the novel normalization was used. Interestingly, miR-21, 
Table IV. miRNAs significantly changed in SCC of the tongue (using data normalized with the novel method).

\begin{tabular}{|c|c|c|c|}
\hline Up-regulated miRNA & p-value & Down-regulated miRNA & p-value ${ }^{a}$ \\
\hline hsa-miR-424 & $3,49 \mathrm{E}-06$ & hsa-miR-617 & $1,23 \mathrm{E}-10$ \\
\hline hsa-miR-21 & $5,55 \mathrm{E}-06$ & hsa-miR-29b-2* & $1,57 \mathrm{E}-07$ \\
\hline hsa-miR-1301 & $6,86 \mathrm{E}-06$ & hsa-miR-132* & $1,74 \mathrm{E}-06$ \\
\hline hsa-miR-7 & $2,85 \mathrm{E}-05$ & hsa-miR-548b-5p & $3,78 \mathrm{E}-05$ \\
\hline hsa-miR-21 ${ }^{*}$ & $3,74 \mathrm{E}-05$ & hsa-miR-509-5p & $8,77 \mathrm{E}-05$ \\
\hline hsa-miR-142-5p & $6,31 \mathrm{E}-05$ & hsa-miR-22* & 2,32E-04 \\
\hline hsa-miR-105 & $7,74 \mathrm{E}-05$ & hsa-miR-99a & $8,76 \mathrm{E}-04$ \\
\hline hsa-miR-142-3p & $1,04 \mathrm{E}-04$ & hsa-miR-203 & $1,39 \mathrm{E}-03$ \\
\hline hsa-miR-146b-3p & $1,34 \mathrm{E}-04$ & hsa-miR-1261 & $1,72 \mathrm{E}-03$ \\
\hline hsa-miR-659 & 2,39E-04 & hsa-miR-34a & $1,72 \mathrm{E}-03$ \\
\hline hsa-miR-361-3p & $3,52 \mathrm{E}-04$ & hsa-miR-30b* & $4,16 \mathrm{E}-03$ \\
\hline hsa-miR-665 & $4,13 \mathrm{E}-04$ & hsa-miR-629* & $5,60 \mathrm{E}-03$ \\
\hline hsa-miR-146b-5p & $6,64 \mathrm{E}-04$ & hsa-miR-29b-1* & 7,07E-03 \\
\hline hsa-miR-206 & $1,27 \mathrm{E}-03$ & hsa-miR-382 & 7,10E-03 \\
\hline hsa-miR-1204 & $2,06 \mathrm{E}-03$ & hsa-miR-644 & $1,12 \mathrm{E}-02$ \\
\hline hsa-miR-1290 & $2,16 \mathrm{E}-03$ & hsa-miR-510 & $1,18 \mathrm{E}-02$ \\
\hline hsa-miR-500 & $2,25 \mathrm{E}-03$ & hsa-miR-375 & $1,29 \mathrm{E}-02$ \\
\hline hsa-miR-936 & $3,13 \mathrm{E}-03$ & hsa-miR-1226 & $1,36 \mathrm{E}-02$ \\
\hline hsa-miR-195* & $3,96 \mathrm{E}-03$ & hsa-miR-100 & $2,20 \mathrm{E}-02$ \\
\hline hsa-miR-583 & $4,45 \mathrm{E}-03$ & hsa-let-7c & $3,35 \mathrm{E}-02$ \\
\hline hsa-miR-1303 & $8,95 \mathrm{E}-03$ & hsa-miR-876-3p & $3,68 \mathrm{E}-02$ \\
\hline hsa-miR-611 & $9,32 \mathrm{E}-03$ & & \\
\hline hsa-miR-146a & $1,29 \mathrm{E}-02$ & & \\
\hline hsa-miR-1304 & $1,30 \mathrm{E}-02$ & & \\
\hline hsa-miR-1298 & $1,70 \mathrm{E}-02$ & & \\
\hline hsa-miR-523 b & $1,93 \mathrm{E}-02$ & & \\
\hline hsa-miR-1300 & $1,93 \mathrm{E}-02$ & & \\
\hline hsa-miR-192 & $2,68 \mathrm{E}-02$ & & \\
\hline hsa-miR-765 & 2,97E-02 & & \\
\hline hsa-miR-625 & $3,23 \mathrm{E}-02$ & & \\
\hline hsa-miR-622 & $3,80 \mathrm{E}-02$ & & \\
\hline hsa-miR-212 & 4,74E-02 & & \\
\hline hsa-miR-124* & $4,80 \mathrm{E}-02$ & & \\
\hline
\end{tabular}

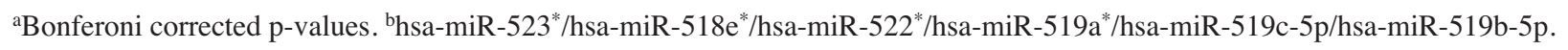

previously shown to be deregulated in tongue SCC, was identified by the novel approach only, and not by the traditional approach.

Confirmation in fresh-frozen sample using q-PCR. Three miRNA detected as significantly differentially expressed when the novel normalization was used (miR-424, miR-21 and miR-203) were confirmed in a set of five fresh-frozen tongue tumours with corresponding paired normal tissue using q-PCR. miR-21 and miR-424 showed an average up-regulation of 4.3- and 4.0-fold, respectively in tumour samples whereas miR-203 was down-regulated on average 2.6-fold in tumour samples, in concordance with the array data (Fig. 3).

Dependency of miRNA sequence. Fixation in formalin modifies RNA and it is known that both sequence identity and length of the RNA will affect modification rate and extent of degradation. miRNAs are all of similar size but differ in their sequence and it was therefore of interest to investigate the relationship between the size of the storage time effect (b-coefficients) and the sequence of the miRNA. 


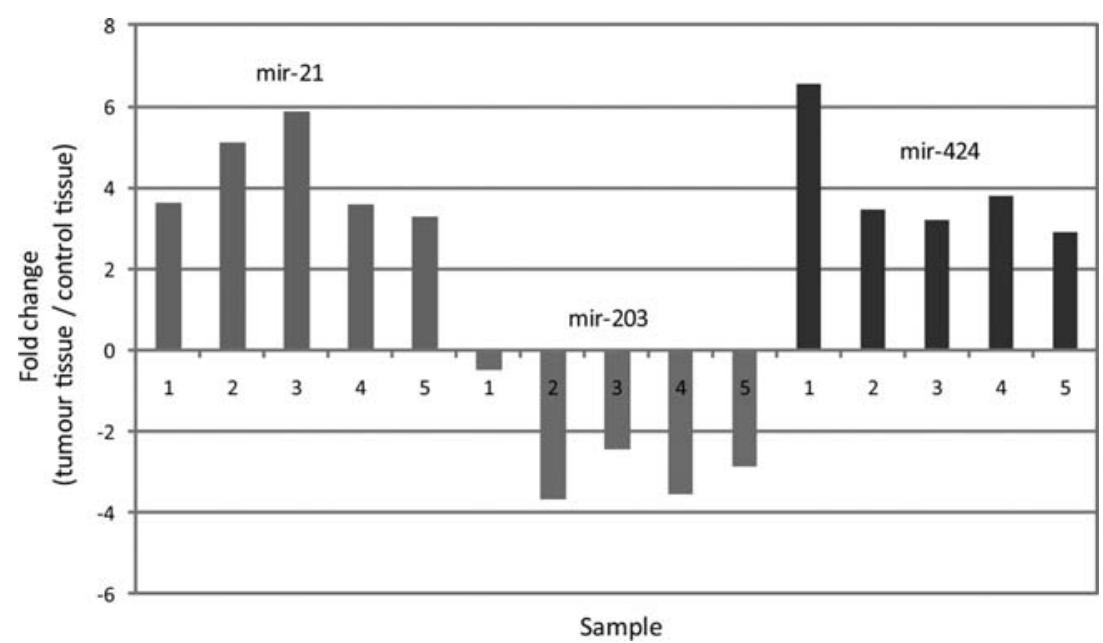

Figure 3. q-PCR data for miR-21, miR-203 and miR-424. The fold change (y-axis) of the three miRNAs in five pairs (x-axis) of FF tongue tumour samples and normal tissue (control tissue) from the same patient.

A

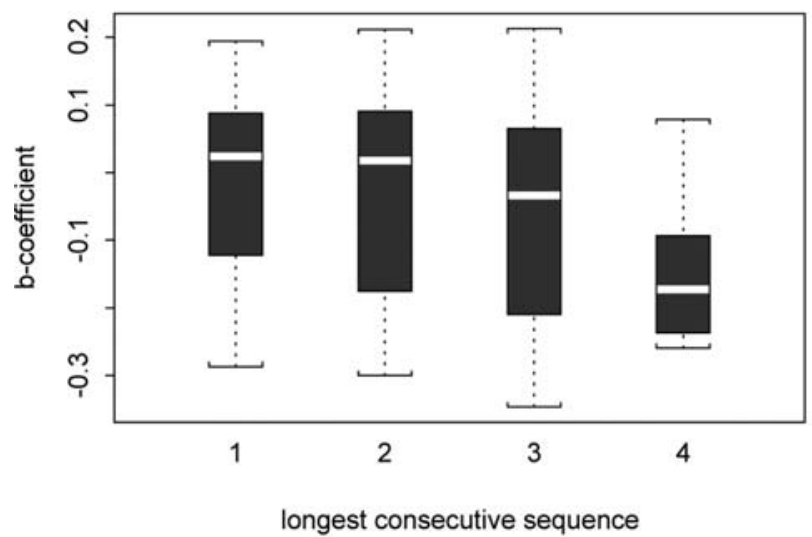

G

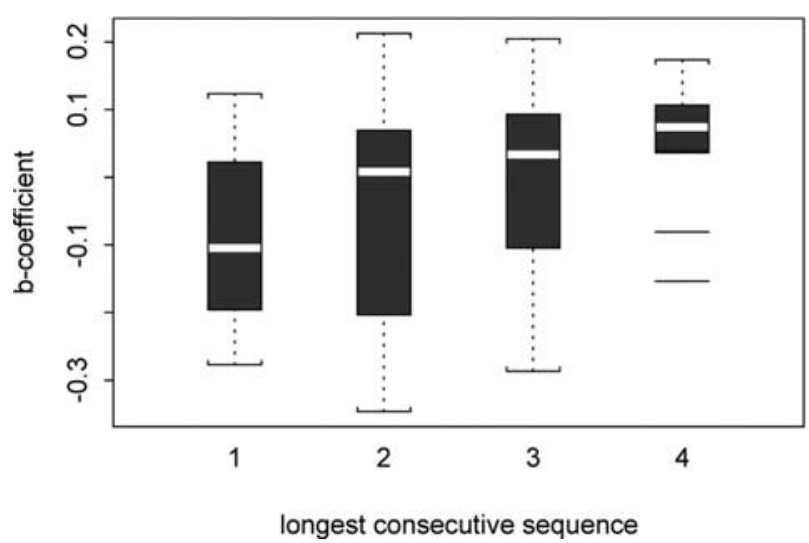

C

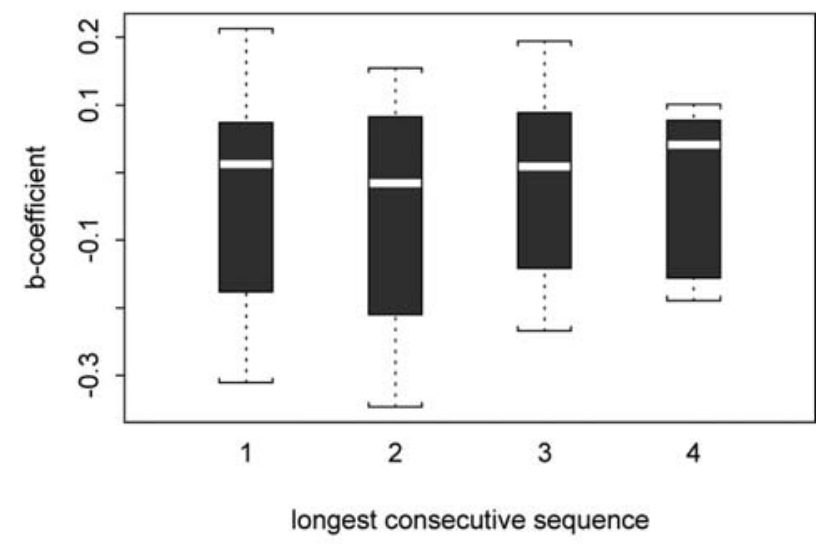

U

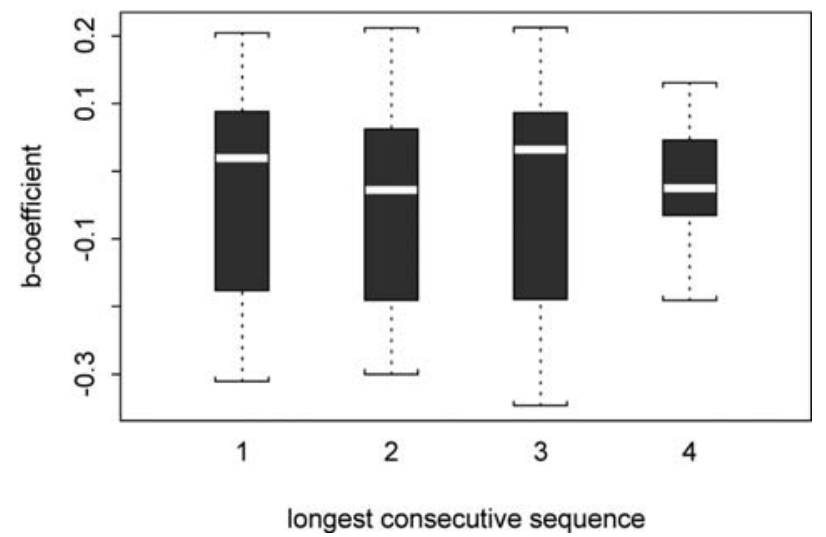

Figure 4. Boxplots of the relationship between number of consecutive A, C, G, or $\mathrm{U}$ in the miRNA sequence and the b-coefficients. Only miRNA with an A-value $>8$ were included and only the longest consecutive sequence in each miRNA was considered. The miRNAs were merged into four classes according to the length of the sequence; longest sequence $\leq 1,2,3$ or $\geq 4$.

The total number of $\mathrm{A}, \mathrm{C}, \mathrm{G}$ or $\mathrm{U}$ in the sequence of a miRNA was not significantly associated with the storage time effect, whereas the number of consecutive As and Gs were $(\mathrm{p}=0.004$ and $\mathrm{p}=0.0001)$ (Fig. 4).

\section{Discussion}

MiRNAs have been proposed to be more stable in FFPE tissue than mRNAs. When measuring the expression of 836 miRNAs 
in 29 FFPE tongue samples of varying ages we observed a variation (bias) correlating to storage time. By performing an additional correction step this bias was overcome, and a large number of miRNAs identified as being significantly differentially expressed between tumour and control samples. miR-21, one of the most studied miRNAs and frequently up-regulated in a number of malignancies $(25,26)$, was significantly upregulated and miR-203, which is epithelium specific and suggested to promote epithelial differentiation $(27,28)$ was significantly down-regulated, correlating well with previous studies of SCC of the head and neck. A novel finding was the consistent upregulation of miR-424 in tumour samples. Upregulation of this miRNA has not been seen before in SCC of the head and neck but has been implicated in a number of malignancies including ovarian cancer (29), non-small cell lung carcinoma (30) and kidney cancer (31). One potential miR-424 target, ITGAV, has previously been seen to significantly correlate with differentiation and metastasis in laryngeal and hypopharyngeal carcinomas $(29,32)$.

A number of studies investigating the usefulness of miRNA expression analysis in FFPE tissue have been performed, and many show good correlation between FFPE and FF samples (16-18). Some, however, have noted a decrease in miRNAs detected with time in storage. This could indicate degradation of miRNA over time or a decrease in labelling efficiency due to increased recovery of short non-specific RNA (17-19). One study found a number of miRNAs to be up regulated in FFPE but not FF samples, which was hypothesised to be caused by non-specific binding of fragmented mRNA and immature miRNA to the array. Another study found a third of the studied miRNAs to be $>2$-fold higher or lower in FFPE compared to FF samples (19). No previous study has, however, reported as large storage time-dependent bias as observed in the current study, which could be explained by the fact that few studies have covered as many samples spread over such a long time period before. In a previous study on mRNA expression, covering almost the same FFPE samples, we did not see the same effect of storage time (33). For mRNA arrays, non-specific binding is less of a problem as longer probes can be designed. We therefore hypothesize that for the miRNA array the combination of non-specific hybridization and degradation caused the storage time dependency seen in the array data.

In conclusion, by studying a series of FFPE human cancer samples, we found that age of samples influences results of miRNA profiling and reduces the efficiency in detecting differentially expressed miRNAs. Data still contain large amounts of information and because of the great potential of the use of FFPE samples, research on methods for handling the storage time effects are of great importance. In our hands a linear regression model proved useful for overcoming the storage time effect enabling identification of miRNAs differentially expressed in tongue tumours.

\section{Acknowledgments}

This study was supported by grants from the Cancer Research Foundation in Northern Sweden, Lion's Cancer Research Foundation, Umeå University, the Swedish Cancer Society Contract number 080371 and Västerbotten County Council.

\section{References}

1. Lee RC, Feinbaum RL and Ambros V: The C. elegans heterochronic gene lin-4 encodes small RNAs with antisense complementarity to lin-14. Cell 75: 843-854, 1993.

2. Wightman B, Ha I and Ruvkun G: Posttranscriptional regulation of the heterochronic gene lin-14 by lin-4 mediates temporal pattern formation in C. elegans. Cell 75: 855-862, 1993.

3. Griffiths-Jones S, Saini HK, van Dongen S, Enright AJ: miRBase: tools for microRNA genomics. Nucleic Acids Res 36: D154-D158, 2008.

4. Kloosterman WP and Plasterk RH: The diverse functions of microRNAs in animal development and disease. Dev Cell 11: 441-450, 2006.

5. Ruan K, Fang X and Ouyang G: MicroRNAs: Novel regulators in the hallmarks of human cancer. Cancer Lett 285: 116-126, 2009.

6. Lewis BP, Burge CB and Bartel DP: Conserved seed pairing, often flanked by adenosines, indicates that thousands of human genes are microRNA targets. Cell 120: 15-20, 2005.

7. Lu J, Getz G, Miska EA, et al: MicroRNA expression profiles classify human cancers. Nature 435: 834-838, 2005.

8. Rosenfeld N, Aharonov R, Meiri E, et al: MicroRNAs accurately identify cancer tissue origin. Nat Biotechnol 26: 462-469, 2008.

9. Baek D, Villen J, Shin C, Camargo FD, Gygi SP and Bartel DP: The impact of microRNAs on protein output. Nature 455: 64-71, 2008.

10. Lim LP, Lau NC, Garrett-Engele P, et al: Microarray analysis shows that some microRNAs downregulate large numbers of target mRNAs. Nature 433: 769-773, 2005.

11. Selbach M, Schwanhausser B, Thierfelder N, Fang Z, Khanin R and Rajewsky N: Widespread changes in protein synthesis induced by microRNAs. Nature 455: 58-63, 2008.

12. Slodkowska EA and Ross JS: MammaPrint 70-gene signature: another milestone in personalized medical care for breast cancer patients. Expert Rev Mol Diagn 9: 417-422, 2009.

13. Lewis F, Maughan NJ, Smith V, Hillan K and Quirke P: Unlocking the archive-gene expression in paraffin-embedded tissue. J Pathol 195: 66-71, 2001.

14. Masuda N, Ohnishi T, Kawamoto S, Monden M and Okubo K: Analysis of chemical modification of RNA from formalin-fixed samples and optimization of molecular biology applications for such samples. Nucleic Acids Res 27: 4436-4443, 1999.

15. von Ahlfen S, Missel A, Bendrat K and Schlumpberger M: Determinants of RNA quality from FFPE samples. PLoS One 2: e 1261, 2007.

16. Li J, Smyth P, Flavin R, et al: Comparison of miRNA expression patterns using total RNA extracted from matched samples of formalin-fixed paraffin-embedded (FFPE) cells and snap frozen cells. BMC Biotechnol 7: 36, 2008.

17. Szafranska AE, Davison TS, Shingara J, et al: Accurate molecular characterization of formalin-fixed, paraffin-embedded tissues by microRNA expression profiling. J Mol Diagn 10: 415-423, 2008.

18. Hasemeier B, Christgen M, Kreipe H and Lehmann U: Reliable microRNA profiling in routinely processed formalin-fixed paraffin-embedded breast cancer specimens using fluorescence labelled bead technology. BMC Biotechnol 8: 90, 2008.

19. Siebolts U, Varnholt H, Drebber U, Dienes HP, Wickenhauser C and Odenthal $\mathrm{M}$ : Tissues from routine pathology archives are suitable for microRNA analyses by quantitative PCR. J Clin Pathol 62: 84-88, 2009.

20. Dudoit S, Yang YH, Callow MJ and Speed TP: Statistical methods for identifying genes with differential expression in replicated cDNA microarray experiments. Statistical Sinica 12: 111-139, 2002.

21. Bolstad BM, Irizarry BA, Astrand M and Speed TP: A comparison of normalization methods for high density oligonucleotide array data based on variance and bias. Bioinformatics 19: 185-193, 2003.

22. Eisen MB, Spellman PT, Brown PO and Botstein D: Cluster analysis and display of genome-wide expression patterns. Proc Natl Acad Sci USA 95: 14863-14868, 1998.

23. Raychaudhuri S, Stuart JM and Altman RB: Principal components analysis to summarize microarray experiments: application to sporulation time series. Pac Symp Biocomput: 455-466, 2000.

24. Baldi P and Long AD: A Bayesian framework for the analysis of microarray expression data: regularized t-test and statistical inferences of gene changes. Bioinformatics 17: 509-519, 2001. 
25. Yan LX, Huang XF, Shao Q, et al: MicroRNA miR-21 overexpression in human breast cancer is associated with advanced clinical stage, lymph node metastasis and patient poor prognosis. RNA 14: 2348-2360, 2008.

26. Liu X, Jiang L, Wang A, Yu J, Shi F and Zhou X: MicroRNA138 suppresses invasion and promotes apoptosis in head and neck squamous cell carcinoma cell lines, Cancer Lett 286: 217-222, 2009.

27. Lena AM, Shalom-Feuerstein R, Rivetti di Val Cervo P, et al: Candi. miR-203 represses 'stemness' by repressing DeltaNp63. Cell Death Differ 15: 1187-1195, 2008.

28. Yi R, Poy MN, Stoffel M and Fuchs E: A skin microRNA promotes differentiation by repressing 'stemness'. Nature 452: 225-229, 2008.

29. Dahiya N, Sherman-Baust CA, Wang TL, et al: MicroRNA expression and identification of putative miRNA targets in ovarian cancer. PLoS One 3: e2436, 2008.
30. Rui W, Bing F, Hai-Zhu S, Wei D and Long-Bang C: Identification of microRNA profiles in docetaxel-resistant human nonsmall cell lung carcinoma cells (SPC-A1). J Cell Mol Med 14: 206-214, 2010.

31. Petillo D, Kort EJ, Anema J, Furge KA, Yang XJ and Teh BT: MicroRNA profiling of human kidney cancer subtypes. Int J Oncol 35: 109-114, 2009.

32. Lu JG, Sun YN, Wang C, Jin de J and Liu M: Role of the alpha $\mathrm{v}$-integrin subunit in cell proliferation, apoptosis and tumor metastasis of laryngeal and hypopharyngeal squamous cell carcinomas: a clinical and in vitro investigation. Eur Arch Otorhinolaryngol 266: 89-96, 2009.

33. Rentoft M, Laurell G, Coates PJ, Sjostrom B and Nylander K: Gene expression profiling of archival tongue squamous cell carcinomas provides sub-classification based on DNA repair genes. Int J Oncol 35: 1321-1330, 2009. 